\title{
High-Flux Beam Source for Cold, Slow Atoms or Molecules
}

\section{Citation}

Maxwell, S. E., N. Brahms, R. deCarvalho, D. R. Glenn, J. S. Helton, S. V. Nguyen, D. Patterson, J. Petricka, D. DeMille, and J. M. Doyle. 2005. High-Flux Beam Source for Cold, Slow Atoms or Molecules. Physical Review Letters 95, no. 17. doi:10.1103/physrevlett.95.173201.

\section{Published Version}

doi:10.1103/PhysRevLett.95.173201

\section{Permanent link}

http://nrs.harvard.edu/urn-3:HUL.InstRepos:27867250

\section{Terms of Use}

This article was downloaded from Harvard University's DASH repository, and is made available under the terms and conditions applicable to Other Posted Material, as set forth at http:// nrs.harvard.edu/urn-3:HUL.InstRepos:dash.current.terms-of-use\#LAA

\section{Share Your Story}

The Harvard community has made this article openly available.

Please share how this access benefits you. Submit a story.

\section{Accessibility}




\title{
High-Flux Beam Source for Cold, Slow Atoms or Molecules
}

\author{
S. E. Maxwell, ${ }^{1}$ N. Brahms, ${ }^{1}$ R. deCarvalho, ${ }^{1}$ D. R. Glenn, ${ }^{2}$ J. S. Helton, ${ }^{3}$ S. V. Nguyen, ${ }^{1}$ D. Patterson,,${ }^{1}$ J. Petricka, ${ }^{2}$ \\ D. DeMille, ${ }^{2}$ and J.M. Doyle ${ }^{1}$ \\ ${ }^{1}$ Department of Physics, Harvard University, Cambridge, Massachusetts 02138, USA \\ ${ }^{2}$ Department of Physics, Yale University, New Haven, Connecticut 06520, USA \\ ${ }^{3}$ Department of Physics, Massachusetts Institute of Technology, Cambridge, Massachusetts 02139, USA
}

(Received 8 June 2005; published 17 October 2005)

\begin{abstract}
We demonstrate and characterize a high-flux beam source for cold, slow atoms or molecules. The desired species is vaporized using laser ablation, then cooled by thermalization in a cryogenic cell of buffer gas. The beam is formed by particles exiting a hole in the buffer gas cell. We characterize the properties of the beam (flux, forward velocity, temperature) for both an atom $(\mathrm{Na})$ and a molecule $(\mathrm{PbO})$ under varying buffer gas density, and discuss conditions for optimizing these beam parameters. Our source compares favorably to existing techniques of beam formation, for a variety of applications.
\end{abstract}

PACS numbers: $39.10 .+\mathrm{j}$

Cold, slow beams of atoms or molecules are of wide utility. A common use of such beams is as a source for loading into traps, where the particles can be further cooled and manipulated, e.g., to create Bose condensates or Fermi degenerate gases. Because the number of trapped particles is typically limited by the characteristics of the initial beam (flux, forward velocity, temperature, etc.), significant effort has been put into developing improved atomic beam sources [1-7]. Work on developing cold molecular sources has recently been a particularly active field of research [813]. As with atoms, one of the aims is to produce quantum degenerate gases, including those comprising strongly interacting electric dipoles [14-16].

For the purposes of loading traps, an ideal source would produce a large flux of any atom or molecule at temperatures less than the depth of the trap, $T_{t}$. For most currently used trap technologies, $T_{t} \lesssim 1 \mathrm{~K}$. For species amenable to laser cooling, this temperature is within the capture range of a magneto-optical trap [17]. For paramagnetic atoms and molecules, strong magnetic traps can have depths exceeding $1 \mathrm{~K}$ [18]. For polar molecules, electric fieldbased traps can reach similar depths $[19,20]$.

We describe here a simple, robust source that can operate with nearly any atomic or molecular species, and which produces a beam at high flux with translational and rotational temperatures near $1 \mathrm{~K}$. We demonstrate this source for both an atom $(\mathrm{Na})$ and a polar molecule $(\mathrm{PbO})$, and discuss the different regimes of beam formation. Our analysis makes it possible to estimate and optimize various characteristics of the beam source for general use. We believe this provides an attractive alternative to many beam techniques now in use.

A simple outline of the operation of our source is as follows. Atoms or molecules of the desired species, $A$, are first vaporized by laser ablation. This produces $N$ particles of $A$ per pulse, at a high temperature $T_{i}$. The ablation takes place inside a cryogenic cell filled with $\mathrm{He}$ buffer gas at low temperature $T_{b}$ and density $n_{\mathrm{He}}$. After a characteristic number of collisions $\mathcal{N}$, the translational temperature $T$ of $A$ comes arbitrarily close to equilibrium with the buffer gas, such that $T \approx(1+\epsilon) T_{b}$ when $\mathcal{N}=-\kappa \ln \left(\epsilon T_{b} / T_{i}\right)$ [21]. [Here $\kappa \equiv\left(m_{A}+m_{\mathrm{He}}\right)^{2} /\left(2 m_{A} m_{\mathrm{He}}\right)$, and $m_{\mathrm{He}}\left(m_{A}\right)$ is the mass of $\mathrm{He}(A)$.] Rotational degrees of freedom are also cooled during these collisions. By allowing both $\mathrm{He}$ and $A$ particles to exit the cell via a small hole, a beam of $A$ is formed. The beam persists for a duration given by the diffusion lifetime of $A$ in the cell, which is limited by the sticking of $A$ particles upon contact with the cell walls.

The number of cold particles of $A$ in the beam is determined by both $n_{\mathrm{He}}$ and the cell geometry. During thermalization, a particle of $A$ typically travels a distance $R \approx \mathcal{N} /\left(n_{\mathrm{He}} \sigma_{t}\right)$, where $\sigma_{t}$ is a thermally averaged cross section for elastic collisions. Hence, for a cell with distance $R_{h}$ from the ablation point to the hole, the particles of $A$ will be efficiently thermalized before exiting the hole only if $R<R_{h}$. In addition, the purely geometric probability for a particle of $A$ to escape in the beam is governed by the ratio $d / R_{h}$, where $d$ is the diameter of the exit hole.

The forward velocity, $v_{f}$, of the thermalized beam of $A$ particles also depends on both $n_{\mathrm{He}}$ and $d$. Specifically, $v_{f}$ is determined by the ratio $d / \lambda$, where $\lambda=1 /\left(n_{\mathrm{He}} \sigma_{c}\right)$ is the mean free path of $A$ particles in the cell; here $\sigma_{c}$ is the elastic cross section for cold $A$-He collisions. In the effusive limit $(\lambda \gg d), v_{f}$ will be given approximately by the thermal velocity of cold $A$ particles, i.e., $v_{f} \approx v_{A} \equiv$ $\sqrt{2 k_{B} T_{b} / m_{A}}$. By contrast, when $\lambda \ll d$ the $A$ particles will become entrained in the outward flow of $\mathrm{He}$, so that $v_{f} \approx v_{\mathrm{He}} \equiv \sqrt{2 k_{B} T_{b} / m_{\mathrm{He}}}$. Since $m_{A} \gg m_{\mathrm{He}}$ for most species of interest, $v_{f}$ is much smaller in the effusive limit than for an entrained beam.

Note that the conditions for efficient thermalization and for a slow beam are in conflict. Thermalization is most efficient for $n_{\mathrm{He}}$ above a threshold value, but effusive flow demands that $n_{\mathrm{He}}$ be less than a typically different threshold. The highest flux of cold, slow A particles is obtained 
when $R_{h}$ and $d$ are chosen so that these thresholds in buffer gas density coincide (namely, when $R_{h} / d \approx \mathcal{N} \sigma_{c} / \sigma_{t}$ ), and $n_{\mathrm{He}}$ is set at this common threshold value $\left(n_{\mathrm{He}}^{-1}=\right.$ $\left.d \sigma_{c}=R_{h} \sigma_{t}\right)$.

A schematic of the beam setup is given in Fig. 1. The buffer gas cell is a brass box $\sim 10 \mathrm{~cm}$ on edge. The cell is mounted in vacuum, with the top face attached to the cold plate of a liquid He cryostat. A $0.8 \mathrm{~mm}$ long exit hole with $d=3 \mathrm{~mm}$ is centered on one side face. The bottom and other side faces are covered with windows for optical access. Several ablation targets are mounted on the top face at $R_{h} \approx 6 \mathrm{~cm}$. The ablation light consists of laser pulses of $\sim 5 \mathrm{~ns}$ duration, with energy $\sim 15 \mathrm{~mJ}$, focused to a spot size $\lesssim 1 \mathrm{~mm}$, at a wavelength of $532 \mathrm{~nm}$. The ablation laser is typically fired at $10 \mathrm{~Hz}$ repetition rate. We produce $\mathrm{Na}$ atoms with sodium metal or $\mathrm{NaCl}$ targets, and $\mathrm{PbO}$ molecules with a vacuum hot-pressed $\mathrm{PbO}$ target. With our ablation conditions, typically $T_{i} \approx 1000 \mathrm{~K}$ for both species.

Buffer gas continuously flows into the cell through a narrow tube that is thermally anchored to the cold plate. This ensures that the buffer gas is at the temperature of the cold plate upon entry to the cell. The cell walls, and thus the He gas, are typically at $T_{b}=5 \mathrm{~K}$. Helium in the cell must be replenished as it flows continuously through the exit hole. A calibrated metering valve at room temperature is used to control the flow into the cell, and hence the density, of the buffer gas. We determine $n_{\mathrm{He}}$ to within a factor of 2. Good vacuum is maintained in the beam region by means of a coconut charcoal sorption pump with a pumping speed of $\sim 10001 \mathrm{~s}^{-1}$.

For a typical elastic cross section $\sigma_{c} \approx 3 \times 10^{15} \mathrm{~cm}^{-3}$, the crossover between effusive and entrained flow of $A$ particles-i.e., the condition $d=\lambda$-occurs for $n_{\mathrm{He}}=$ $n_{c} \approx 10^{15} \mathrm{~cm}^{-3}$. This should be compared to the density $n_{\mathrm{He}}=n_{t}$ required for full thermalization of $A$ particles -

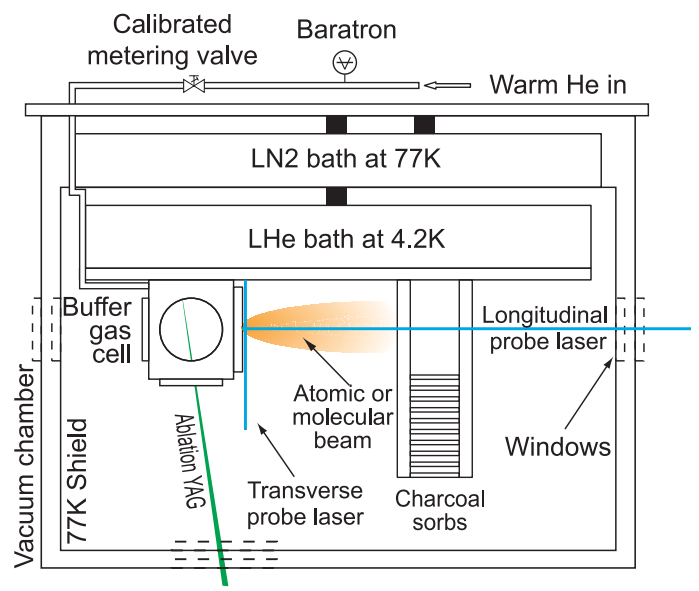

FIG. 1 (color online). A schematic of the beam apparatus. When detecting fluorescence, a lens (not shown) collimates a fraction of the fluorescence light and directs it out a window to a PMT. i.e., such that $R \approx \mathcal{N} /\left(n_{\mathrm{He}} \sigma_{t}\right)=R_{h}$. Assuming $\sigma_{t} \approx \sigma_{c}$, we find that for $\mathrm{Na}$ in our cell, $n_{t} \approx n_{c}$. Thus, it should be anticipated that our cell is near the optimal geometry for producing a maximal flux of slow, cold $\mathrm{Na}$. By contrast, the larger mass of $\mathrm{PbO}$ makes $\mathcal{N}$ much larger than for $\mathrm{Na}$, implying that our cell geometry is not optimal for $\mathrm{PbO}$. We characterize the beam source for both species within a range of densities around the anticipated optimal condition for Na, namely $n_{\mathrm{He}} \approx(0.2-5) \times 10^{15} \mathrm{~cm}^{-3}$.

The beam source is monitored using laser spectroscopy. Doppler shifts and widths of the spectra are used to determine beam velocity profiles. Signal size and timing yield the column density and particle dynamics. To measure the longitudinal (transverse) velocity profile, a probe laser beam is sent collinear with (perpendicular to) the molecular beam. For $\mathrm{Na}$, a third probe beam monitors atoms inside the cell.

We monitor $\mathrm{Na}$ atoms via absorption of a probe laser tuned to the $3 s_{1 / 2} \rightarrow 3 p_{3 / 2}$ transition at wavelength $\lambda_{\mathrm{Na}}=$ $589 \mathrm{~nm}$. The probe laser frequency is continuously scanned over the entire absorption profile (a range of $\sim 1 \mathrm{GHz}$ ), at a rate of $1 \mathrm{kHz}$, resulting in several complete spectral profiles of the $\mathrm{Na}$ atomic beam for each ablation pulse. The relatively slow scan of the laser ensures that we are sensitive only to nearly thermalized $\mathrm{Na}$ atoms; hot atoms traverse the detection region before a scan is complete.

$\mathrm{PbO}$ is monitored via laser-induced fluorescence. This probe laser is tuned to the $X\left(v^{\prime \prime}=0\right) \rightarrow B\left(v^{\prime}=5\right)$ transition at $\lambda_{\mathrm{PbO}, e}=406 \mathrm{~nm}$. Fluorescence is detected using a photomultiplier tube (PMT), with interference and colored glass filters to selectively observe the $B\left(v^{\prime}=5\right) \rightarrow$ $X\left(v^{\prime \prime}=4\right)$ transition at $\lambda_{\mathrm{PbO}, f}=460 \mathrm{~nm}$. A shutter with an opening time of $\sim 7 \mathrm{~ms}$ is used to shield the PMT from the initial glow following each ablation pulse. Again, this technique ensures that only slow-moving molecules are detected. The signal is averaged over several (typically 10) shots of the ablation laser with the probe laser frequency fixed. A single spectral scan consists of frequency points separated by $10-30 \mathrm{MHz}$ across a span of up to several GHz. Our quantitative determination of molecule number from fluorescence counts includes estimates of the detection efficiency and branching fraction for the detected transition [22]. We estimate the error in our absolute number measurement of $\mathrm{PbO}$ to be less than a factor of 2 .

For $\mathrm{Na}$, the in-cell probe beam was used to determine both $N$ and $\sigma_{c}$. We find $N_{\mathrm{Na}} \approx 10^{14} /$ pulse for both the $\mathrm{Na}$ and $\mathrm{NaCl}$ targets. We measure diffusion lifetimes of $\tau[\mathrm{ms}] \approx 4 \times 10^{-15} \times n_{\mathrm{He}}\left[\mathrm{cm}^{-3}\right]$. From this we infer $\sigma_{c} \approx 3 \times 10^{-15} \mathrm{~cm}^{2}$. For $\mathrm{PbO}$, previous work has measured an ablation yield of $\approx 10^{12} /$ pulse [23], and our measurements with an in-cell probe indicate a comparable yield.

In Fig. 2 we plot the number, $N_{h}$, of thermalized particles of species $A$ exiting the hole as a function of $n_{\mathrm{He}}$. We 


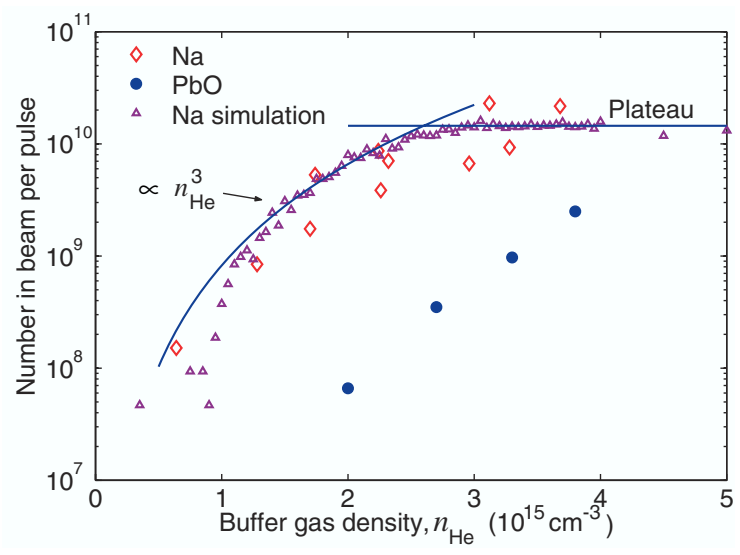

FIG. 2 (color online). The number of cold $A$ particles, $N_{h}$, that emerge in the beam as a function of $n_{\mathrm{He}}$. Curves with specific functional forms have been inserted to show the different scaling regimes. The intersection of the curves corresponds to the condition $R=R_{h}$, where a typical $A$ particle is thermalized just as it reaches the hole.

also show the results of a Monte Carlo simulation of the beam formation process. The condition for full thermalization is apparent in both the experimental and the simulated data for $\mathrm{Na}$, while as expected we do not appear to reach the condition of full thermalization for $\mathrm{PbO}$.

In the $\mathrm{Na}$ data and simulations, we find that $N_{h}$ increases rapidly (approximately $\propto n_{\mathrm{He}}^{3}$ ) up to a critical value of $n_{\mathrm{He}}$, above which $N_{h}$ is roughly constant at its maximum value $N_{h \text {,max }}$. The low-density scaling is consistent with a simple picture in which $A$ particles are distributed uniformly over a volume of characteristic length $L_{t} \approx \mathcal{N} \lambda$, when they have thermalized to near $T_{b}$. (This broad distribution arises, in our simulations of the thermalization process, from the spreads both in the number of collisions required to thermalize and in the free path between collisions.) At high density, the condition $N_{h} \simeq N_{h \text {, max }}$ arises in our simulations when $R<R_{h}$ for essentially all $A$ particles. In this regime, the fraction of $A$ particles escaping, $f_{\max }=$ $N_{h, \max } / N$, is given roughly by the area of the hole to the area of a hemisphere at radius $R_{h}$, i.e., $f_{\max } \approx d_{h}^{2} /\left(8 R_{h}^{2}\right)$. In our geometry, $f_{\max } \approx 3 \times 10^{-4}$.

The simulated beam data in Fig. 2 are scaled to match the experimental $\mathrm{Na}$ data by adjusting the values of $N_{\mathrm{Na}}$ and $\sigma_{t}$. The resulting value, $N_{\mathrm{Na}} \approx 5 \times 10^{13}$, is in reasonable agreement with the determination from the in-cell probe. The fitted thermalization cross section, $\sigma_{t} \sim 1 \times$ $10^{-15} \mathrm{~cm}^{2}$, is somewhat smaller than $\sigma_{c}$. This is reasonable, since elastic cross sections are typically smaller at higher collision energies [24].

Figure 3 shows the average forward velocity, $v_{f}$, of the beams of $A$ particles as $n_{\mathrm{He}}$ is varied. For both $\mathrm{Na}$ and $\mathrm{PbO}$, the data show a nearly linear increase of $v_{f}$ with $n_{\mathrm{He}}$, with the velocity of the lighter species always larger. This behavior is consistent with the following simple picture.

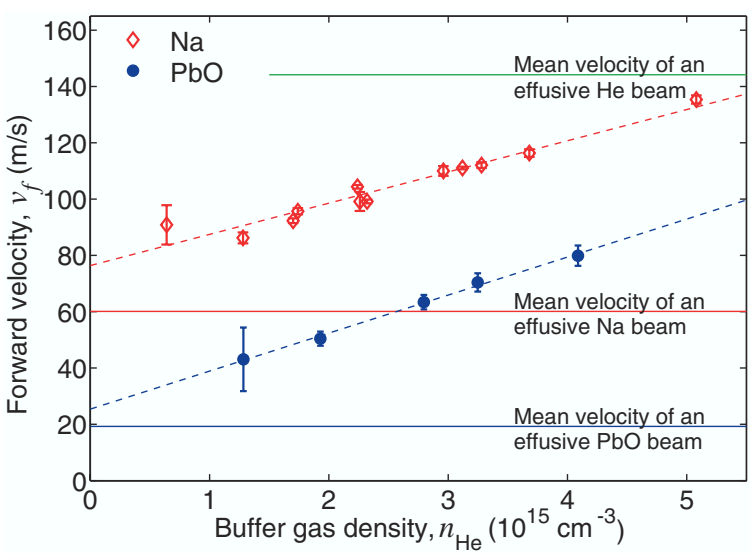

FIG. 3 (color online). $\mathrm{PbO}$ and $\mathrm{Na}$ beam mean forward velocities, $v_{f}$, as a function of $n_{\mathrm{He}}$. Extrapolation of the data to zero buffer gas density is illustrated by best-fit lines (dashed lines).

A slowly moving particle of $A$ takes a time $T_{e}$ to exit the hole, where $T_{e} \sim d / v_{A}$. During this time, it undergoes $N_{e}$ collisions with fast, primarily forward-moving He atoms, where $N_{e} \sim n_{\mathrm{He}} \sigma_{c} v_{\mathrm{He}} T_{e}$. Each collision imparts a momentum transfer $\Delta p_{A} \sim m_{\mathrm{He}} v_{\mathrm{He}}$. This results in a net velocity boost $\Delta v_{A}$, given by $\Delta v_{A} \sim v_{A} d / \lambda \propto n_{\mathrm{He}}$. This picture should be roughly valid for densities below the regime of full entrainment, where $v_{f} \sim v_{\mathrm{He}}$. The velocities we measure for $\mathrm{Na}$ are approximately reproduced by modeling of the beam formation process with our measured value of $\sigma_{c}$.

This picture also predicts that the behavior of $v_{f}$, when extrapolated to $n_{\mathrm{He}}=0$, should yield the velocity of an effusive beam of $A$ particles at temperature $T_{b}$. To make this comparison, it is critical to note that our detection technique is sensitive to molecules within a roughly cylindrical volume, of diameter $D_{d}$, and with length $L$ extending from the exit hole. Under our conditions, where $D_{d} \sim d<$ $L$, it can be shown that an effusive beam will exhibit a velocity distribution close to $f(v) \propto v^{2} e^{-m_{A} v^{2} /\left(2 k_{B} T_{b}\right)}$ and a mean velocity $\bar{v}_{\text {eff }} \approx 1.13 v_{A}$. Our extrapolated data are within $\sim 25 \%$ of this prediction for both species.

Figure 4 shows the temperature of the beams vs $n_{\mathrm{He}}$, as measured by the velocity spreads in the longitudinal and transverse directions, as well as the rotational population distribution (for $\mathrm{PbO}$ ). For transverse temperature we fit to a distribution of the form $f_{t}(v) \propto e^{-m_{A} v^{2} /\left(2 k_{B} T\right)}$. For longitudinal temperature we use $f_{l}(v) \propto e^{-m_{A}\left(v-v_{l}\right)^{2} /\left(2 k_{B} T\right)}$. Fits of the longitudinal data to the effusive distribution $f_{l, \text { eff }}(v) \propto v^{2} e^{-m_{s} v^{2} /\left(2 k_{B} T\right)}$ were poor, consistent with the partial entrainment of $A$ in the helium flow. For the rotational temperature, we use Clebsch-Gordan and HönlLondon factors to determine the relationship between fluorescence intensity and initial state population for various rotational lines. The ratio of initial state populations for $2-$ 3 rotational lines determine the temperature corresponding to a Boltzmann distribution. Our data indicate complete 


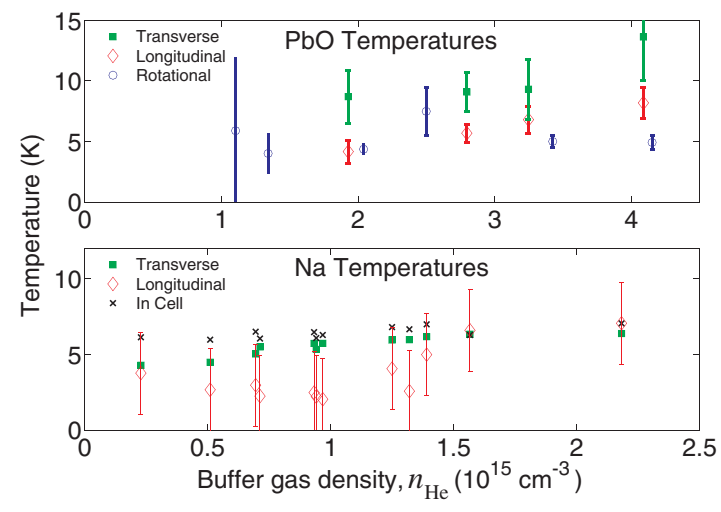

FIG. 4 (color online). $\mathrm{PbO}$ and $\mathrm{Na}$ temperatures as a function of $n_{\mathrm{He}}$ as determined by fits to spectral profiles. "Transverse," "longitudinal," and "in cell" refer to the location of the probe laser (see Fig. 1). Typical errors for the transverse and in cell $\mathrm{Na}$ temperatures are comparable to the marker size.

thermalization of all detected particles. Note that we observe no additional cooling below $T_{b}$, as would be expected for entrainment in a fully supersonic He flow.

The beam source described here can be readily adapted to the needs of a wide range of experiments. For example, it can be used for a wide variety of species with performance similar to that described here. The total flux depends linearly on the ablation yield $N$, which for any given target is difficult to predict a priori. However, for nearly every species we have tried (here and in many related experiments), it has been possible to achieve large values of $N$ by a suitable choice of precursor material. For example, under operating conditions similar to those used here, we have obtained $N=10^{12}-10^{14}$ for a variety of metal atoms and $N=10^{11}-10^{13}$ for many species of diatomic molecules (including radicals) [25-28]. In addition - subject to limitations of cooling power or gas load - the source could be run at lower temperatures, higher repetition rates, or with higher extraction efficiency (e.g., by using several separated exit holes). Our discussion of the beam formation mechanism makes it straightforward to determine the effects of such changes.

A key possible improvement to the source would be the addition of a guide, either magnetic (for paramagnetic species) or electric (for polar molecules). In both cases, $\mathrm{He}$ is unaffected by the guide potential and will exit through the sides of the guide, allowing extraction of the $A$ beam into a region of ultrahigh vacuum. Under the conditions described here, this beam source could be used to load a peak flux of up to $2 \times 10^{11} \mathrm{~s}^{-1} \mathrm{Na}$ atoms into a simple permanent magnet guide such as the type described in Ref. [7]. An electrostatic guide such as that described in Ref. [12] could be loaded with a peak flux of $\sim 10^{9} \mathrm{~s}^{-1} \mathrm{PbO}$ molecules in the $J=1$ rotational state, adequate for loading into a microwave [19] or electrostatic [29] trap. The high fluxes from our source could result in substantial improvements in atomic and molecular trapping experiments that depend on large initial numbers.

We acknowledge support from the NSF under Grants No. DMR-0325580 and No. PHY-0071311, the Army Research Office, the W.M. Keck Foundation, and the David and Lucile Packard Foundation.

[1] Z. T. Lu et al., Phys. Rev. Lett. 77, 3331 (1996).

[2] C. Slowe, L. Vernac, and L. V. Hau, physics/0407040.

[3] J. Schoser et al., Phys. Rev. A 66, 023410 (2002).

[4] K. Dieckmann, R. J.C. Spreeuw, M. Weidemuller, and J. T. M. Walraven, Phys. Rev. A 58, 3891 (1998).

[5] P. Cren et al., Eur. Phys. J. D 20, 107 (2002).

[6] R. S. Conroy et al., Opt. Commun. 226, 259 (2003).

[7] E. Nikitin et al., Phys. Rev. A 68, 023403 (2003).

[8] M. Gupta and D. Herschbach, J. Phys. Chem. A 105, 1626 (2001).

[9] H.L. Bethlem, G. Berden, and G. Meijer, Phys. Rev. Lett. 83, 1558 (1999).

[10] M. R. Tarbutt et al., Phys. Rev. Lett. 92, 173002 (2004).

[11] M.S. Elioff, J. J. Valentini, and D. W. Chandler, Science 302, 1940 (2003).

[12] S. A. Rangwala et al., Phys. Rev. A 67, 043406 (2003).

[13] R. Fulton, A.I. Bishop, and P.F. Barker, Phys. Rev. Lett. 93, 243004 (2004).

[14] L. Santos, G. V. Shlyapnikov, P. Zoller, and M. Lewenstein, Phys. Rev. Lett. 85, 1791 (2000).

[15] M. A. Baranov, M.S. Mar'enko, Val. S. Rychkov, and G. V. Shlyapnikov, Phys. Rev. A 66, 013606 (2002).

[16] M. Baranov et al., Phys. Scr. T102, 74 (2002).

[17] E. L. Raab et al., Phys. Rev. Lett. 59, 2631 (1987).

[18] J. G. E. Harris et al., Rev. Sci. Instrum. 75, 17 (2004).

[19] D. DeMille, D. R. Glenn, and J. Petricka, Eur. Phys. J. D 31, 375 (2004).

[20] H. Bethlem et al., Nature (London) 406, 491 (2000).

[21] R. deCarvalho et al., Eur. Phys. J. D 7, 289 (1999).

[22] E. A. Dorko et al., Chem. Phys. 102, 349 (1986).

[23] D. Egorov et al., Phys. Rev. A 63, 030501(R) (2001).

[24] L. Landau and E. Lifshitz, Quantum Mechanics (Nonrelativistic Theory), Course of Theoretical Physics Vol. 3 (Pergamon Press, New York, 1977), 3rd revised ed.

[25] J. Kim et al., Phys. Rev. Lett. 78, 3665 (1997).

[26] B. Friedrich, J. Weinstein, R. deCarvalho, and J. Doyle, J. Chem. Phys. 110, 2376 (1999).

[27] B.S. Zhao et al., Rev. Sci. Instrum. 75, 146 (2004).

[28] C. I. Hancox et al., Nature (London) 431, 281 (2004).

[29] H. L. Bethlem et al., Phys. Rev. A 65, 053416 (2002). 